\title{
Verifying environmental relationships
}

\author{
RICHARD C. SHERMAN, CELIA OLIVER, and WILLIAM TITUS \\ Miami University, Oxford, Ohio 45056
}

\begin{abstract}
In two experiments, subjects first learned the locations of objects in a $21 \times 21 \mathrm{ft}$ room and then were timed as they verified, from memory, statements about the spatial relationships of the objects to certain features (e.g., the outside walls, the center of the room, subdividing partitions). The statements were of the form " $X$ is (not) close to (far from) F" in Experiment 1 and " $\mathrm{X}$ is farther from (closer to) $\mathrm{F}$ than $\mathrm{Y}$ is" in Experiment 2, where $\mathrm{X}$ and $\mathrm{Y}$ are specific objects and $F$ is a feature. While some of the latencies varied directly with the magnitudes of the distances between objects and features named in the sentences, there were several instances in which sentences associated with equivalent distances led to different latencies or sentences associated with different distances led to equivalent latencies. The possible cognitive operations underlying the latency pattern are discussed.
\end{abstract}

The purpose of the present research is to investigate how people accomplish the task of verifying, from memory, statements about the spatial relations among objects in the environment. Consider, for example, the common situation in which one person makes a verbal request to another for information about an environment, such as, "Is the new supermarket closer to downtown than the old one?" or "Is your office far from the building entrance?" The stated relationships in these requests concern the relative proximity ("close," "closer," "far," "farther") of specific objects (supermarkets, office) to particular environmental features or aspects of the environment that serve as locational referents ("downtown," "building entrance"). In order to respond to this common type of question, the listener must somehow verify the relationships conveyed verbally against his or her spatial knowledge of the environment.

There are a number of possible operations that might be involved in verifying environmental relationships such as these. One type of process might operate directly on specific distance magnitudes retrieved from memory. For example, the truth or falseness of a sentence like "X is close to F," where $X$ is a specific object and F is some environmental feature, might be determined by retrieving an analog representation of the X-F distance magnitude and then comparing this with an appropriate standard or cutoff for "close." Retrieved distances judged to be smaller than the cutoff would lead to "true" responses, and distances larger than the cutoff would produce "false" responses. For sentences of the type " $\mathrm{X}$ is closer to $\mathrm{F}$ than $\mathrm{Y}$ is," representations of the $X-F$ and $Y-F$ distances might be retrieved and compared to assess their relative magnitude. The outcome $\mathrm{X}-\mathrm{F}<\mathrm{Y}-\mathrm{F}$ would result in a "true" decision, whereas

Portions of this research were presented to the Midwestern Psychological Association Convention, May 1979. Requests for reprints should be addressed to the first author, Department of Psychology, Miami University, Oxford, Ohio 45056.
X-F $>$ Y-F would lead to "false." Thus, the verification process might rely on retrieval and assessment operations similar to those investigated in studies of spatial imagery (e.g., Kosslyn, Ball, \& Reiser, 1978) and subjective magnitude comparisons (e.g., Holyoak, 1977; Moyer, 1973; Paivio, 1975). Most of the recent research that has focused specifically on representations of environment information would seem to be consistent with this view. These studies have tended to emphasize the perceptual and sensorimotor qualities of environmental knowledge as revealed in estimates of remembered bearing and distance (e.g., Baird, 1979; Herman, Kail; \& Siegel, 1979; Kosslyn, Pick, \& Fariello, 1974; Kozlowski \& Bryant, 1977; Sherman, Croxton, \& Giovannatto, 1979; Sherman, Croxton, \& Smith, 1979).

However, other types of operations are also plausible. Although continuous or analog environmental distance information may be available in memory, it might not be operated upon directly in the verification of the type of relational statements being considered here. One possibility is that the task requires the retrieval of discrete distance codes that correspond only to the level of generality conveyed by the sentence. These codes might be of the same general form proposed by Banks, Fujii, and Kayra-Stuart (1976) and Banks and Root (1979) for subjective comparisons along other magnitude dimensions. Banks and his colleagues have argued that magnitude information on any dimension is made available initially as crude semantic codes that are determined by a generation process sensitive to whether stimuli fall above or below an implicit criterion. The comparison operation is then performed on these codes, rather than on the precise magnitudes that give rise to them. Here, it might be proposed that the sentence " $\mathrm{X}$ is close to $\mathrm{F}$ " would be verified by transforming "close" into the instruction code "SHORT" and then comparing this with the retrieved semantic code associated with the X-F distance. The available code for a magnitude less than the implicit criterion would also be SHORT, and 
the match of codes would lead to a "true" response. The retrieved distance code "LONG" would not match the instruction code and would result in a "false" response. A similar code-matching process can be derived for sentences of the type " $\mathrm{X}$ is closer to $\mathrm{F}$ than $\mathrm{Y}$ is."

Although the semantic code idea is no less plausible for environmental distance than it is for other dimensions, the verification of relational statements may involve more than a simple comparison of semantic codes. It can be noted that a sentence like " $\mathrm{X}$ is close to F" asserts a relational proposition. It is plausible that the spatial knowledge necessary for the verification is made available in the same propositional format. The sentence and knowledge codes are then compared to determine if they "mean" the same thing. The operations for carrying out this comparison might therefore follow the general sentence verification principles proposed by authors such as Carpenter and Just (1975) and Clark and Chase (1972, 1974). For instance, in the Clark and Chase model, the truth of an assertion is computed by sequentially comparing each component in the sentence representation (i.e., argument, predicate, polarity marker) with its counterpart in the memory representation. A binary truth index, initially set to TRUE, is changed to its opposite value upon each mismatch of components, and its final state determines the assessed truth value of the sentence.

In the present situation, the memory code for a short distance between $X$ and $F$ might be available as $\operatorname{AFF}[\operatorname{CLOSE}(\mathrm{X}, \mathrm{F})]$, where AFF symbolizes a polarity marker asserting the relationship as being true. A sentence like " $\mathrm{X}$ is not far from $\mathrm{F}$ " might be represented as NEG $[F A R(X, F)]$. Comparisons of the components in the two representations would result in the final state of the truth index being TRUE, since there are two mismatches (CLOSE vs. FAR and AFF vs. NEG). Likewise, the memory code for the relative distances of two objects, $\mathrm{X}$ and $\mathrm{Y}$, to a common feature, F, might be available as AFF[CLOSER TO $F(X, Y)]$. The sentence " $X$ is farther from $F$ than $Y$ is," represented as AFF[FARTHER FROM F(X,Y)], would be judged false, since the relational components (CLOSER vs. FARTHER) mismatch. These propositional representations of environmental knowledge might be constructed ad hoc from crude semantic codes (Banks et al., 1976) or from distance information retrieved by a scanning operation (Kosslyn et al., 1978). Alternatively, they may be stored in memory as previous interpretations of precise magnitudes and are retrieved directly.

It is not possible to assess the role of the various operations outlined above on the basis of previous research. Subjective magnitude comparison studies, for instance, have focused on dimensions other than environmental distance, such as remembered object size, the relative magnitudes of digits, or the relative loudness of tones. Spatial imagery work involving distance has generally been restricted to diagrammatic stimuli. The sentence verification research, particularly that of Clark and Chase $(1972,1974)$, has perhaps come the closest to addressing directly the topic of verifying spatial relationships. Even here, however, spatial information was presented only in simple diagrams or pictures, and the sentences to be verified followed almost immediately. In contrast, most environmental knowledge involves the integration of fragmentary information obtained over relatively longer periods of time and from multiple perspectives (cf. Allen, Siegel, \& Rosinski, 1978; Evans \& Pezdek, 1980; Neisser, 1976; Sadalla, Staplin, \& Burroughs, 1979).

The two experiments reported here required subjects to acquire and use "true" environmental spatial knowledge. In both experiments, subjects first learned the layout of a small, novel environment and then were timed as they verified, from memory, sentences about certain spatial relationships. The learning task in both experiments was designed to parallel three common aspects of how people acquire spatial information: (1) the association of verbal labels with objects and their locations, (2) the necessity of moving through the environment to gain complete spatial information, and (3) the lack of a specific anticipation as to subsequent uses of the information. In Experiment 1, the sentences that were verified contained the relational terms "close" and "far," and distance magnitudes were allowed to vary with both the truth value and relational form of the sentences. In Experiment 2, the sentences contained the comparatives "closer" and "farther," and the distance magnitudes were held constant across truth value and relational form.

In Experiment 1, true close and false far sentences were constructed to refer to the same relatively small distances. Likewise, true far and false close sentences referred to the same relatively larger distances. It was assumed that different verification operations might yield correspondingly different latency patterns in these particular instances. In general, the contribution of magnitude or image-based processes to the latencies should be tied to the specific sizes of the distances involved. For example, the influence of image-scanning operations (cf. Kosslyn et al., 1978) might be revealed by longer overall latencies for the sentences pertaining to longer distances (true far and false close) than for those pertaining to shorter distances (true close and false far). Magnitude comparison operations might contribute latencies that decrease as the discrepancy between the retrieved distance magnitude and implicit cutoff increases. In the present case, this discrepancy would most likely be larger for false sentences (e.g., assertions that a short distance is "far" or a longer distance is "close") than for true sentences, although the effect of the difference is difficult to specify, since the cutoff values are unknown.

On the other hand, processes that are based on comparing semantic codes or propositional representations 
need not produce latencies that are linked to the precise magnitudes of the distances in any direct way. For example, a proposition-comparison process might be reflected in latencies that follow a "componentmismatch" rule. Sentences involving more mismatches would take longer (regardless of the precise distance) than those involving fewer mismatches. Thus, one possibility is that sentences might differ in overall verification latency, even though the distance relationships are identical. The relative speed would be determined by the form of the propositional representation available for the verification (CLOSE or FAR) and the resulting number of mismatches between sentence and memory components. Consider, for example, the following schematic relationship between an object $(\mathrm{X})$ and two features $\left(F\right.$ and $\left.F^{\prime}\right)$ : FX-..-F'. The memory code for this relationship might be structured either in terms of $\mathrm{X}$ being close to $\mathrm{F}$ (proximity) or in terms of $\mathrm{X}$ being far from $F^{\prime}$ (separation). A sentence asserting that $X$ is close to $F^{\prime}$ would produce a feature mismatch in the first case, but two in the second (feature and relation). Although the decision outcome is the same, the number of mismatches differs, and so might the latency pattern.

The various relationships between sentence form, truth value, distance magnitude, and the number and type of potential mismatches for Experiment 1 are summarized in Table 1.

\section{EXPERIMENT 1}

\section{Method}

Subjects. Sixteen undergraduates, eight men and eight women, participated to obtain research credit for introductory psychology courses.

Stimulus environment. A diagram of the experimental environment is shown in Figure $1 .^{1}$ The environment consisted of a $21-\mathrm{ft}(6.4-\mathrm{m})$ square room uniformly illuminated by fluorescent ceiling panels. The outside edges of the environment were defined by the room's walls, which were covered with white cloth to ensure uniformity of appearance. Four "districts" of equal size and shape were defined within the environment by using four $6 \times 6 \mathrm{ft}(1.8 \mathrm{~m} \times 1.8 \mathrm{~m})$ screens. Two of the screens were transparent and two were opaque. In the sentences presented to subjects, the screens were referred to as "clear" and "black" barriers, respectively. The screens were arranged so that they also defined a central area of the environment that provided common access to all four districts. Each district contained three stimulus locations, which were marked by identical wood

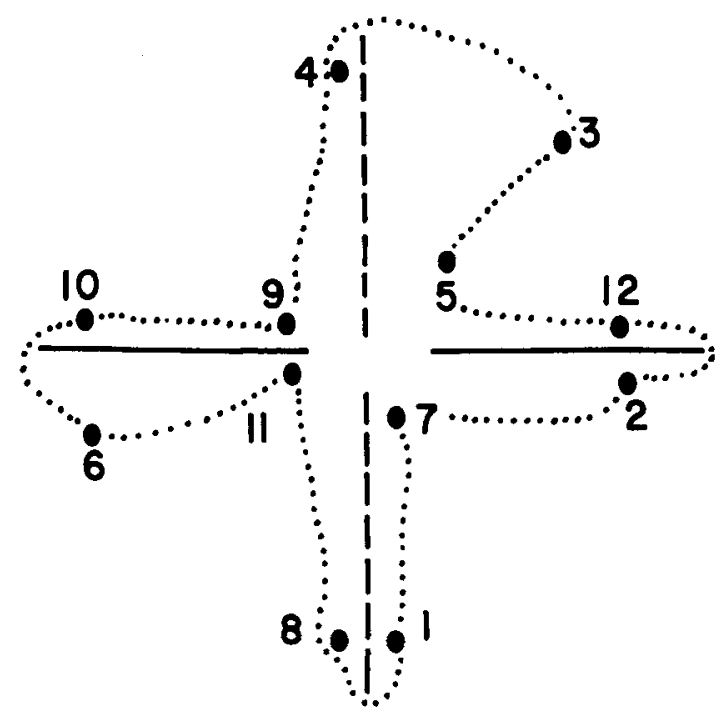

Figure 1. Diagram of the stimulus environment. Solid lines indicate opaque barriers, and dashed lines indicate transparent barriers. Path taken by subjects during initial learning phase is shown by dots. Numbering of locations is arbitrary.

pedestals 29 in. $(51 \mathrm{~cm})$ high. Thus, the environment contained 12 specific objects that could be spatially related to the screens, the center of the room, and the outside walls. The objects were positioned so that subsets of them were in close proximity to each of these reference features.

Location names. The acquisition phase of the experiment was described to subjects as being analogous to learning the locations and names of buildings on a university campus. Accordingly, 12 surnames were selected to meet the following criteria: (1) All were two syllables and approximately the same length, (2) each name began with a different letter of the alphabet, (3) no two names rhymed, (4) all names were of uniform ethnicity, (5) none were names of buildings actually on campus, and (6) none were names of prominent figures known to subjects. The names selected were Bower, Chapman, Edwards, Felton, Garrett, Hanley, Jenkin, Landis, Merrill, Penner, Sperling, and Wilcox. Four different random assignments of names to locations were used in the experiment, with equal numbers of subjects (two men and two women) learning each assignment. The names were printed on pieces of paper and placed on the tops of the location pedestals described above. They were hidden from view by identical cardboard cylinders $10 \times 10$ in. $(25 \times 25 \mathrm{~cm})$, painted flat black and closed at one end.

Procedure. Each subject participated individually. Before entering the experimental environment, the subject was told that the object of the study was to find out more about how people learn new environments. It was explained that the subject's

Table 1

Distance Magnitudes and Numbers of Component Mismatches for Sentences in Experiment 1

\begin{tabular}{lcll} 
& & & Component Mismatches \\
\cline { 3 - 4 } Sentence Type & Distance & \multicolumn{1}{c}{ Proximity Code } & Separation Code \\
\hline True Close & Small & 0 & 2 (Relation + Feature) \\
False Close & Large & 1 (Feature) & 1 (Relation) \\
True Far & Large & 2(Relation + Feature) & 1 (Feature) \\
False Far & Small & 1 (Relation) & 2 (Polarity Marker + Relation) \\
True Not-Close & Large & 2 (Polarity Marker + Feature) & 3 (Polarity Marker + Relation + Feature) \\
False Not-Close & Small & 1 (Polarity Marker) & 2 (Polarity Marker + Feature) \\
True Not-Far & Small & 2(Polarity Marker + Relation) & 1 (Polarity Marker) \\
False Not-Far & Large & 3 (Polarity Marker + Relation + Feature) &
\end{tabular}


task would be to learn where everything was in a new environment "in relation to everything else." No mention was made of the verification task. The subject was then blindfolded, led into the environment, and positioned at the center.

The acquisition phase of the experiment was divided into five parts. First, the blindfold was removed and the subject was given one orientation trial in which he or she was escorted to each pedestal and shown the name under its cardboard cylinder. The order in which the names were shown was determined randomly for each subject, with the constraint that each successive location be in a different district. Part 2 consisted of a series of learning trials in which the subject guessed the name at each location while being escorted along a predetermined route. After each guess, the subject was allowed to lift the cylinder and see the correct name. Although the subject always traveled from location to location along the same route (see Figure 1), the starting point and direction of travel were changed on every trial to prevent the subject's learning simply the sequence of names, rather than their locations. The starting points were chosen so that: (1) on the first trial, the starting point was in the district diagonally opposite that of the last location seen on the orientation trial, and (2) successive starting points were in different districts and each was in a district different from the ending point of the preceding trial. This procedure was continued until the subject completed two trials without making any errors.

The remaining parts of the acquisition phase were designed to ensure the thoroughness of the subject's knowledge of the environment. In Part 3, the experimenter verbally gave the subject a location name and asked to be taken to that location. This was repeated for each of the remaining locations in a random order with the constraint that successive locations be in different districts. The subject was not restricted in movement during this or the remaining parts of the acquisition phase. In Part 4 , the experimenter verbally gave the subject four names, each from a different district and randomly ordered, and asked to be taken to each location in turn. This was repeated five more times, until each of the locations had been used twice. Finally, in Part 5, the subject was taken to each district in a random order and asked to identify all the locations within it. If a mistake was made during these last three tasks, the subject was allowed to make corrections. Mistakes after the last learning trial were very rare, however. Only seven errors were observed for all 16 subjects.

The verification phase of the experiment was conducted in a different room and began approximately $5-10 \mathrm{~min}$ after the acquisition phase. It was explained to the subject that the task was to decide as quickly as possible whether statements about spatial relationships in the environment he or she had just learned were best described as "true" or "false." The subject was encouraged to be as fast as possible while also giving the "best" (i.e., the "most descriptive") response. Four practice sentences were then presented, to familiarize the subject with the general procedure and with the form of the stimulus sentences.

The set of stimulus sentences consisted of all possible combinations of the 12 location names, the relations "close" and "far," in both positive and negative form, and the features "clear barrier," "black barrier," "center," and "outside" (e.g. "Hanley is not close to the center"). The sentences were presented on a VT11 display monitor controlled by a PDP-11 computer. Each sentence appeared on the display screen $1 \mathrm{sec}$ after the subject depressed the "ready" button on a response box and remained on until the subject responded by pressing either a "true" or "false" button. The computer recorded the time interval in milliseconds between the onset of the stimulus and the subject's response. The subject kept both hands on the box, using either thumb for depressing the "ready" button and the right and left forefingers for depressing the appropriate response button. For half the subjects, "true" was on the right side of the box, and for half, it was on the left, counterbalanced across subject and location name assignment condition. Each subject received the sentences in a different random order.

\section{Results and Discussion}

The a priori truth value of each sentence was set by considering the actual relationship between the location and feature contained in the sentence. For the purpose of analysis, Locations 5, 7,9, and 11 (see Figure 1) were considered to be close to the center and far from the outside, and all other locations were considered to be close to the outside and far from the center. Similarly, Locations 1, 7, 8, and 4 were treated as close to clear barriers and Locations 2, 12, 9, 10, and 11 as close to black barriers. Locations 1,8 , and 4 were treated as far from black barriers, and 2,12 , and 10 as far from clear barriers. ${ }^{2}$ The several locations within each of the above groups provided replications of true and false sentences for each relation-feature combination. Each subject's response latencies to the several sentences in each category were averaged together. A latency was included in a given average only if the subject's response ("true" or "false") matched the a prior truth value of the sentence. The overall proportion of responses that did not match the a priori truth value was $8.5 \%$, which is comparable to the error rates in previous sentence verification studies (e.g., Clark \& Chase, 1972, Experiment 2; Clark and Chase, 1974, Experiment 2) and indicates that subjects agreed with the "close" and "far" designations of the objects. Errors were moderately correlated with latencies $(r=.54)$.

A truth by feature by relation by polarity analysis of variance performed on the averaged latencies indicated significant main effects for all four variables. True sentences were verified more quickly $(3,227 \mathrm{msec})$ than false sentences $(3,339 \mathrm{msec}) \quad[F(1,15)=9.15$, $p<.01]$. Sentences containing "close" were verified faster $(3,145 \mathrm{msec})$ than those with "far" $(3,422 \mathrm{msec})$ $[F(1,15)=21.11, p<.001]$. Negative sentences took longer to verify $(3,519 \mathrm{msec})$ than affirmative sentences $(3,048 \mathrm{msec})[\mathrm{F}(1,15)=20.39, \mathrm{p}<.001]$. Finally, a main effect of feature type was found $[F(3,45)=3.69$, $\mathrm{p}<.02]$, such that the longer barrier sentences took significantly more time than center-outside sentences $[F(1,15)=4.82, p<.05]$. Center and outside sentences did not differ significantly from each other, nor did clear barrier and black barrier sentences. The latter finding indicates that additional time is needed to process the barrier information, probably because of the somewhat longer sentences involved. However, the verification process seems to be the same for all types of features: No interactions were found between feature type and any other variables.

The result of primary interest was a significant interaction between the truth of sentences, type of relation they contained ("close to" vs. "far from"), and polarity (affirmative vs. negative) $[F(1,15)=28.71$, $\mathrm{p}<.001, \mathrm{MSe}=1.5068 \times 10^{4} \mathrm{~J}$. The mean verification 
Table 2

Mean Latencies ( $L$ ) in Milliseconds and Percent Responses (PR) Not Matching A Priori Truth Values for Sentences in Experiment 1

\begin{tabular}{|c|c|c|c|c|c|c|c|c|}
\hline & \multicolumn{4}{|c|}{ Affirmative Sentences } & \multicolumn{4}{|c|}{ Negative Sentences } \\
\hline & \multicolumn{2}{|c|}{ Close } & \multicolumn{2}{|c|}{ Far } & \multicolumn{2}{|c|}{ Not-Close } & \multicolumn{2}{|c|}{ Not-Far } \\
\hline & $\mathrm{L}$ & PR & $\mathbf{L}$ & PR & $\mathrm{L}$ & PR & $\mathrm{L}$ & PR \\
\hline True & 2732 & 5.7 & 3390 & 13.8 & 3421 & 13.2 & 3366 & 11.5 \\
\hline False & 2990 & 5.3 & 3079 & 8.6 & 3436 & 6.3 & 3852 & 8.4 \\
\hline
\end{tabular}

latencies for this interaction are shown in Table 2 .

Specific comparisons among means within the interaction revealed several differences that appear to be related to the size of the distances associated with the sentences. True close sentences (short distances) were verified significantly ${ }^{3}$ more quickly than false close sentences (longer distances). True far sentences (longer distances) took significantly longer than false far sentences (short distances). For negatives, true not-far relationships (short distances) were verified faster than false not-far (long distances), although the error rates indicate some speed-accuracy tradeoff. Comparing latencies across sentence form, true close verifications (small distances) were made more quickly than true far (larger distances). False not-far sentences (larger distances) were significantly slower than false not-close (small distances).

Considering just these comparisons, the latency pattern suggests a direct, positive relationship between the distance being assessed and the length of time to make a judgment. However, additional comparisons revealed latency differences that did not correspond to specific distance magnitudes in this manner. First, there were several cases in which verification latencies differed significantly, despite the fact that the distance magnitudes were equivalent: true close $<$ false far, true far $>$ false close, and true not-close $<$ false not-far. Second, there were instances in which the distance magnitudes differed but the mean latencies did not: false close vs. false far and true not-close vs. true not-far.

The direct, positive relationship between verification time and distance is consistent with a scanning operation in which greater distances take longer to assess. On the other hand, the several instances in which this relation. ship did not hold suggest that some other process may be operative as well. For example, in all of the comparisons reported above, including those in which the direct distance-latency relationship does not hold, the latency differences correspond to the differences in the number of hypothesized mismatches encountered between sentence propositions and proximity-based memory propositions (cf. Table 1). One possibility, then, is that distance magnitude is retrieved by a scanning operation, but that this information is transformed to a propositional format and the actual verification is carried out by a component-comparison process. This would account for those cases in which latencies differ even though dis- tance magnitudes are the same. It would not, however, explain the instances in which sentences referring to different distances do not differ significantly. Since the numbers of mismatches in these cases are equal, it is tempting to argue that the propositions are either retrieved directly or formed from crude semantic codes, rather than obtained from scanned distance magnitudes. That is, the retrieval operation may be more or less constant across all sentences (as it presumably would be in retrieving propositions or in generating semantic codes), and latency is influenced primarily by the component-matching process. This second possibility is somewhat tenuous, however, since it requires arguing from a null result.

One comparison within the interaction produced an outcome that did not seem to follow either the distance magnitude or the component-mismatch rule. The mean latency for true not-close sentences (longer distances, two mismatches) did not differ significantly from that for false not-close sentences (shorter distances, only one mismatch). An examination of the data separately for the various feature types indicated that this result was due in part to an unusually long (and inexplicable) latency for false sentences of the type " $\mathrm{X}$ is not close to a clear barrier," which averaged $492 \mathrm{msec}$ longer than comparable sentences for other features. This was also the source of the truth main effect reported above. Finally, it must be noted that the false negative sen. tences exhibited a general tendency toward speedaccuracy tradeoff.

The possible role of the proposition-matching process was investigated further in Experiment 2. It was hoped that a somewhat clearer demonstration of its contribution to verification latencies would be obtained by holding constant the potential scanning component discussed above. The nature of the propositional code was also examined in Experiment 2. The overall advantage of close sentences, as well as the specific pattern of latency differences within the Truth by Relation by Polarity interaction, suggests that the propositions made available during the verification task were structured in terms of proximity, rather than separation. Similar subject preferences for one relational form over another have been demonstrated in other contexts (cf. Clark \& Chase, 1972), as well as changes in these preferences as a function of task and stimulus variables (cf. Clark \& Chase, 1974). In general, the proximity of a specific object in the environment to a referent feature is perhaps more naturally useful information than is separation, since it associates the location of the object with a prominent "landmark," narrows the range of possible locations of the object, and therefore makes remembering its location or communicating about it much easier (it is generally easier and more informative to describe where an object is than where it is not). Thus, subjects in Experiment 1 may have followed this general preference in dealing with the specific task at hand. Although this is an admittedly post hoc explanation for the 
preference choice in Experiment 1, it leads to a specific prediction for Experiment 2, in which the sentences to be verified contained the comparative terms "closer" and "farther." The latency results should again follow a component-mismatch rule based on proximity rather than on separation.

\section{EXPERIMENT 2}

In Experiment 2, subjects verified sentences of the form " $X$ is closer to (farther from) $F$ than $Y$ is," where $X$ and $Y$ are specific objects and $F$ is some environmental feature. For each X-F-Y triplet, a "closer" and a "farther" sentence were constructed, and true and false sentences were created by reversing the order of the object names in the sentences. Thus, both true and false versions of any given sentence referred to exactly the same environmental distances. Also, for any given triplet, the distances referred to by a "closer" sentence were identical to those referred to by a "farther" sentence. Operations involving scanning the X-F and Y-F distances and comparing the two specific magnitudes should therefore contribute equally to the verification of all sentences pertaining to the same X-F.Y triplet.

A propositional comparison process would nevertheless still give rise to latency differences based on a component-mismatch rule. If the relationships among elements in an X-F-Y triplet were made available during verification as a proposition of the general form AFF[CLOSER TO $F(X, Y)]$, then comparisons with sentence codes of the same form could produce mismatches between the predicate components, the argument components, or both. The true sentence " $\mathrm{X}$ is closer to $\mathrm{F}$ than $\mathrm{Y}$ is" would be verified more quickly than the false sentence " $Y$ is closer to $F$ than $X$ is," because in the latter, the argument $(\mathrm{Y}, \mathrm{X})$ would mismatch the environmental memory code $(X, Y)$. For sentences containing "farther," the true-false difference would be reversed. The true sentence " $Y$ is farther from $F$ than $X$ is" would produce both predicate and argument mismatches, whereas the false sentence " $\mathrm{X}$ is farther from $F$ than $Y$ is" would yield only a mismatch of predicate components. The general pattern of latencies that would follow from these operations is true closer $<$ false closer $<$ false farther $<$ true farther, assuming the environmental memory codes are consistently structured in terms of proximity (CLOSER) rather than separation (FARTHER). This relational preference would also result in an overall advantage for "closer" sentences.

\section{Method}

Subjects. Twenty undergraduates, 10 men and 10 women, participated to obtain research credit for introductory psychology courses. None of the subjects had served in Experiment 1 .

Procedure. The acquisition phase was identical to that in Experiment 1, including the configuration of the stimulus environment and the location names. As before, four different random assignments of names to locations were used, with equal numbers of subjects assigned to each.

In the verification phase, each subject was told that the task was to decide, as quickly yet accurately as possible, whether statements about spatial relationships in the stimulus environment were true or false. Eight practice sentences were then presented to familiarize subjects with the general form of the statements. The stimulus presentation and response apparatus were the same as those in Experiment 1. Half the subjects in each name-assignment condition operated the "true" button with their right forefinger and half with their left forefinger. Prior to the presentation of each sentence, a message appeared on the screen instructing the subject to press the "ready" button when prepared to receive the sentence. The sentence was displayed $1 \mathrm{sec}$ after the button was depressed, and the verification latency was measured from sentence onset until the subject responded "true" or "false." A message then appeared for $2 \mathrm{sec}$, informing the subject whether the response was correct or incorrect.

The stimulus set consisted of 120 sentences. Each sentence compared two locations from the same district in terms of their relationship to one of the four environmental features (center, outside, clear barrier, black barrier). Half the true sentences for each feature contained "farther from" and half contained "closer to." Equal numbers of false sentences within each of these groups were constructed by reversing the order of names in the sentence. Six pairs of locations were used for center-outside comparisons $(10-9,9-4,5-3,2-7,7-1,6-11)$, making a total of 48 sentences. Nine pairs were used for barrier comparisons (black: 10-4, 4-9, 5-3, 1-12, 2-1, 2-7, 3-7, 8-6, 6-11; clear: $10-9,4-9,4-10,5-3,3-12,2-1,2-7,8-6,6-11$ ), making 72 sentences. Pairs $8-11$ and 5-12 were reserved for practice sentences. The order of presentation of the stimulus set was random within the following constraints: (1) No two consecutive sentences contained the same location name or the same combination of feature and relationship, and (2) no more than four consecutive sentences had the same truth value. Four such orders were used (corresponding to the four name assignments), with equal numbers of subjects assigned to each.

\section{Results and Discussion}

The overall error rate was $11.3 \%$, which is comparable to levels found in previous studies, as is the range of values over specific sentence conditions (cf. Chase \& Clark, 1972, 1974). The errors were highly correlated $(r=.83)$ with the latencies, indicating that the probability of error generally increased with the number of hypothesized operations.

A truth by relation by feature analysis of variance was performed on subjects' correct response latencies, averaged within each of the eight sentence types. The results indicated significant main effects of relation $[F(1,19)=69.90, p<.001]$ and feature $[F(1,19)=$ $12.39, \mathrm{p}<.01]$. Closer sentences $(2,760 \mathrm{msec})$ were verified more quickly than farther sentences $(3,308 \mathrm{msec})$, and center-outside sentences $(2,862 \mathrm{msec})$ were verified faster than barrier sentences $(3,201 \mathrm{mser})$.

A significant Truth by Relation interaction was also obtained $\left[\mathrm{F}(1,19)=15.36, \mathrm{p}<.001, \mathrm{MSe}=5.69 \times 10^{4}\right]$. The cell means are presented in Table 3 . It will be recalled that for sentences of the same relational form, the only difference between true and false versions was the order of objects named in the sentence. Thus, the distances compared or scanned in these cases should 
Table 3

Mean Latencies (L) in Milliseconds and Percent

Error (PE) for Sentences in Experiment 2

\begin{tabular}{lccccc} 
& \multicolumn{2}{c}{ Closer } & & \multicolumn{2}{c}{ Farther } \\
\cline { 2 - 3 } \cline { 5 - 6 } & $\mathrm{L}$ & $\mathrm{PE}$ & & $\mathrm{L}$ & $\mathrm{PE}$ \\
\hline True & 2665 & 4.6 & & 3356 & 20.1 \\
False & 2855 & 6.5 & & 3251 & 14.1 \\
\hline
\end{tabular}

be identical. However, analysis of the Truth by Relation interaction indicated that true closer sentences $(2,665 \mathrm{msec})$ were verified significantly more quickly than false closer sentences $(2,885 \mathrm{msec})$, whereas the reverse was the case for farther sentences $(3,356$ and $3,251 \mathrm{msec}$ for the true and false, respectively).

The interaction pattern is highly consistent with a proposition-comparison process following a componentmismatch rule. For example, true farther sentences would be expected to be verified more slowly than false farther sentences, because in the latter case the only mismatch between the sentence and environmental representations is in the relation conveyed by the sentence. In the former case, both the relation and the order of object names of the sentence mismatch the corresponding components of the memory representation, and these mismatches require extra time-consuming operations. Conversely, true closer sentences would not produce any mismatches, whereas false closer sentences would mismatch in the order of object names. Overall, the ranking of latency magnitudes corresponds to that expected as a result of proximity-based proposition comparisons: true closer $(2,665 \mathrm{msec})<$ false closer $(2,855 \mathrm{msec})<$ false farther $(3,251 \mathrm{msec})<$ true farther $(3,356 \mathrm{msec})$.

\section{GENERAL DISCUSSION}

While some of the latencies in Experiment 1 seemed to vary with the magnitudes of the distances between the objects and features named in the sentences, there were several instances in which sentences associated with equivalent distances led to different latencies or sentences associated with different distances led to equivalent latencies. In Experiment 2, in which distance magnitudes were held constant, verification times were nevertheless observed to vary. Overall, the pattern of latencies in both experiments seems to reflect more than just the operation of magnitude or image-based processes. In particular, the results appear quite consistent with a general proposition-comparison process in which the verification latencies can be roughly indexed by a component-mismatch rule. Thus, in verifying statements that assert propositional relationships among objects in the environment, it seems plausible that the spatial knowledge necessary for the verification is made available in the same propositional format. The exact nature of the source of these codes, however, remains to be determined. As suggested previously, these proposi- tional representations of environmental knowledge might be constructed ad hoc either from scanned distance information or from crude semantic codes of the type proposed by Banks et al. (1976). Alternatively, they might be stored in memory as the result of previous propositional interpretations of precise distance magnitudes.

This conclusion is not meant to imply that there are no situations in which representations of precise distance magnitudes are directly operated upon, but rather that the nature of the task may determine the form of the environmental knowledge that is made available and the nature of the process that is carried out. For instance, if subjects were asked to compare distance magnitudes directly, rather than to verify an assertion about their relative magnitudes, response latencies might well reflect a magnitude comparison process. In a recent study by Baum and Jonides (1979), this appears to be the case (see also Evans \& Pezdek, 1980). Subjects were timed as they decided which of two familiar campus buildings was closer to a third. In general, the latencies were inversely related to the size of the difference between two distances being compared (specifically, there was a positive relationship between latency and the ratio of the smaller distance to the larger). This result seems to be a kind of "symbolic distance effect," a common finding in magnitude comparison studies, and therefore one that might be produced by operations on specific analog magnitudes (Holyoak, 1977; Moyer, 1973; Paivio, 1975) or on semantic magnitude codes (Banks et al., 1976; Banks \& Root, 1979).

In summary, the results of these experiments may illustrate a flexibility in retrieving and using spatial information that perhaps calls for some refinement of the commonly held but narrowly delineated assumption that environmental spatial knowledge is represented in memory as a "cognitive map." An unnecessarily restrictive implication of the term "map" is an image-like format with attendant analog principles governing the retrieval and use of spatial information. As the present data imply, however, alternative principles may also characterize spatial representations. Other recent research has likewise demonstrated the limitations of the map analogy. Stevens and Coupe (1978), for instance, have found that memory for the locations of points (i.e., relative bearing) is influenced by their symbolic hierarchical organization. Similarly, Sadalla et al. (1979) have demonstrated that memory for the length of a traversed route is subject to distortion as a function of the retrievability of incidental route information (names of intersections). Recognition of these organizational and process variables would seem to be essential in furthering our understanding of environmental spatial cognition.

\section{REFERENCES}

Allen, G. L., \& Siegel, A. W., \& Rosinski, R. R. The role of perceptual context in structuring spatial knowledge. Journal 
of Experimental Psychology: Human Learning and Memory, $1978,4,617-630$.

BAIRD, J. C. Studies of the cognitive representation of spatial relations: II. A familiar environment. Journal of Experimental Psychology: General, 1979, 108, 92-98.

Banks, W. P., Fuji, M., \& Kayra-Stuart, F. Semantic congruity effects in comparative judgments of magnitudes of digits. Journal of Experimental Psychology: Human Perception and Performance, 1976, 2, 435-447.

Banks, W. P., \& Root, M. Semantic congruity effects in judgments of loudness. Perception \& Psychophysics, 1979, 26, 133-142.

BaUm, D. R., \& Jonides, J. Cognitive maps: Analysis of comparative judgments of distance. Memory \& Cognition, 1979, 6, 462-468.

Carpenter, P. A., \& Just, M. A. Sentence comprehension: A psycholinguistic processing model of verification. Psychological Review, 1975, 82, 45-73.

Clark, H. H., \& Chase, W. G. On the process of comparing sentences against pictures. Cognitive Psychology, 1972, 3, 472-517.

Clark, H. H., \& Chase, W. G. Perceptual coding strategies in the formation and verification of descriptions. Memory \& Cognition, 1974, 2, 101-111.

Evans, G. W., \& Pezdek, K. Cognitive mapping: Knowledge of real-world distance and location information. Journal of Experimental Psychology: Human Learning and Memory, 1980, 6, 13-24.

Herman, J. F., Kail, R. V., \& Siegel, A. W. Cognitive maps of a college campus: A new look at freshman orientation. Bulletin of the Psychonomic Society, 1979, 13, 183-186.

HOLYOAK, K. J. The form of analog size information in memory. Cognitive Psychology, 1977, 9, 31-51.

Kosslyn, S. M., Ball, T. M., \& Reiser, B. J. Visual images preserve metric information: Evidence from studies of image scanning. Journal of Experimental Psychology: Human Perception and Performance, 1978, 4, 47-60.

Kosslyn, S. M., Pick, H. L., \& F ariello, G. R. Cognitive maps in children and men. Child Development, 1974, 45, 707-716.

Kozlowski, L. T., \& Bryant, K. J. Sense of direction, spatial orientation, and cognitive maps. Journal of Experimental Psychology: Human Perception and Performance, 1977, 3, 590-598.

MOYER, R. S. Comparing objects in memory: Evidence suggesting an internal psychophysics. Perception \& Psychophysics, 1973, $13,180-184$

Neisser, V. Cognition and reality. San Francisco: Freeman, 1976.

Paivio, A. Perceptual comparisons through the mind's eye. Memory \& Cognition, 1975, 3, 635-647.

Sadalla, E. K., Staplin, L. J., \& Burroughs, W. J. Retrieval processes in distance cognition. Memory \& Cognition, 1979, 7, 291-296.

Sherman, R. C., Croxton, J., \& Giovanatto, J. Investigating cognitive representations of spatial relationships. Environment and Behavior, 1979, 11, 209-226.

Sherman, R. C., Croxton, J., \& Smith, M. Movement and structure as determinants of spatial relationships. Journal of Environmental Psychology and Nonverbal Behavior, 1979, 4, 27-39.

Stevens, A., \& Coupe, P. Distortions in judged spatial relations. Cognitive Psychology, 1978, 10, 422-437.

\section{NOTES}

1. The general configuration of the experimental environment is based on a study by Kosslyn et al. (1974), although the exact placement of objects and nature of the task here are quite different.

2. Locations 3 and 5 were in fact equidistant from the two types of barriers, and barrier sentences referring to these locations were not used in the analysis. Location 6 was used in practice sentences.

3. Specific comparisons reported throughout the paper are significant at an experimentwise error rate of .05 by the Dunn test. 